Bender RA, et al. Temporal lobe epilepsy after experimental prolonged febrile seizures: prospective analysis. Brain April 2006;129:911-922). (Respond: Tallie Z Baram MD PhD, Department of Anatomy and Neurobiology and Department of Pediatrics, University of California at Irvine, ZOT 4475, Irvine, CA 92697).

COMMENT. These laboratory studies support the theory that prolonged infantile FS may predispose to later- onset temporal lobe epilepsy (TLE) (Annegers JF, et al. N Engl J Med 1987;316:493-498). The duration of the FS has been shown to correlate with prognosis and the later development of epilepsy and epileptiform EEGs (Millichap JG et al. Neurology $1960 ; 10: 643-653)$. Epilepsy developed in $30 \%$ of patients with prolonged FS ( $>20 \mathrm{~min}$ ) and in only $5 \%$ of those with short FS $(<20 \mathrm{~min})$. The incidence of EEG abnormalities at followup in patients with long and short FS was $36 \%$ and $10 \%$, respectively. In a more recent study, the mean duration of the FS was $100+/-133$ min in patients who later developed TLE and 9+/-19 min in those without TLE at prolonged follow-up (mean 32 years) (Maher J, McLachlan RS. Brain 1995;118:1521-1528). The prevention of febrile seizures, especially the complex type, requires more effective therapeutic intervention.

\title{
ABSENCE EPILEPSY AND MOYAMOYA DISEASE
}

The case of a 6-year-old girl with typical absence epilepsy associated with moyamoya disease (MMD) is reported from the Department of Neurosurgery, Kyoto University Graduate School of Medicine, Japan. The child had suffered from recurrent syncope, approximately five times a day for one year, prior to the onset of absence. At 4 years of age she developed episodes of bilateral weakness of her legs, induced by hyperventilation. An EEG revealed a typical bilateral synchronous $3-\mathrm{Hz}$ spike-and-wave discharge induced by hyperventilation. With high-dose sodium valproate (1800 mg daily) seizures increased in frequency to 10 times daily. An MRI showed a small infarction in the left frontal lobe, and cerebral angiography revealed stenoocclusion of terminal portions of both internal carotid arteries and moyamoya vessels at the base of the brain. Antiplatelet therapy with aspirin was started, and decreased regional cerebral blood flow on SPECT and decreased cerebrovascular reserve were demonstrated in bilateral frontal lobes, presurgically. Following bilateral superficial temporal artery-middle cerebral artery anastomoses, the patient responded to treatment with sodium valproate and aspirin, and she had been free of seizures and transient ischemic attacks for more than 2 years at follow-up. An EEG with hyperventilation after withdrawal of medication showed no bursts of spike-and-wave. This is the first reported case of absence epilepsy associated with MMD. (Kikuta K-I, Takagi Y, Arakawa Y, et al. Absence epilepsy associated with moyamoya disease. Case report. J Neurosurg (4 Suppl Pediatrics) April 2006;104:265-268). (Reprints: Ken-ichiro Kikuta MD PhD, Department of Neurosurgery, Kyoto University Graduate School of Medicine, 54 Kawaharacho, Shogoin, Sakyo-ku, Kyoto 606-8507, Japan).

COMMENT. Moyamoya disease should be considered in the diagnosis of refractory absence epilepsy in Japan, but the prevalence is extremely low. In this case, the preceding syncopal episodes were explained by transient ischemic attacks, and ischemia in the frontal lobes enhanced by hyperventilation was probably related to the occurrence of absence seizures. A possible genetic factor was omitted from the report. 